\title{
Merging Helicobacter pylori eradication and family history-based genetic counseling in patients with gastric cancer: towards an overarching approach in clinical practice
}

\author{
Alexios-Fotios A. Mentis ${ }^{a}$, Theodore Rokkas ${ }^{b}$ \\ Hellenic Pasteur Institute; Henry Dunant Hospital, Athens, Greece
}

Helicobacter pylori (H. pylori) represents the prototype chronic infection of the gastric and potentially the gastrointestinal system, and it is a key model for studying microbially-induced oncogenesis in humans $[1,2]$. H. pylori is now a well-established risk factor for gastric cancer [3], especially through the action of certain microbial genes, such as CagA [4], whereas its eradication has been robustly linked to gastric cancer reduction, especially for those harboring non-atrophic or atrophic gastritis as precancerous lesions [5]. Although perhaps not oft-discussed in daily practice, a family history of gastric cancer is also a major contributing factor [6].

A recent study by Choi et al assessed whether $H$. pylori eradication can reduce the risk for gastric cancer in individuals with a family history of this cancer type in first-degree relatives [7]. The study built upon previous systematic findings that first-degree relatives of patients with gastric cancer present with a higher risk of developing gastric cancer themselves, as evidenced both by $H$. pylori prevalence rates per se and by markers of gastric atrophy and intestinal metaplasia [8]. In that novel study, the authors showed a statistically significant difference in the proportion of patients developing gastric cancer between those treated for $H$. pylori and those who did not receive any treatment $(1.2 \%$ vs. $2.7 \%$, hazard ratio 0.45 , $95 \%$ confidence interval $0.21-0.94 ; \mathrm{P}=0.03$ ) [7]. These results imply that the risk posed by having a family history of gastric cancer can be mitigated by addressing an environmental (in this case, microbial) factor [7]. Nonetheless, they should be interpreted with caution, especially upon considering some methodological caveats in this study, which diminish their broader clinical usefulness (as observed in other $H$. pylorirelated studies [9]). Specifically, the family history of gastric cancer was defined as having at least one first-degree relative with gastric cancer whose diagnosis had been histologically confirmed [7]. However, no search for variants in well-

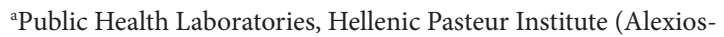
Fotios A. Mentis); ' Gastroenterology Clinic, Henry Dunant Hospital (Theodore Rokkas), Athens, Greece

Conflict of Interest: None

Correspondence to: Dr. Alexios-Fotios A. Mentis, MD, MPH, PgCert, Public Health Laboratories, Hellenic Pasteur Institute, Athens, Greece, e-mail: amentis1@jhu.edu

Received 15 November 2020; accepted 23 November 2020; published online

DOI: https://doi.org/10.20524/aog.2021.0589 established oncogenes and tumor suppressor genes and, in turn, no stratification based on such genetic biomarkers (e.g., E-cadherin or ARID1A) were performed in that study. Doing so would have allowed a more robust genetic confirmation of gastric cancer predisposition [10-12].

Moving forward, a broader question arises, important for clinical practice and clinical cancer genetics counseling: is having a first-degree relative with gastric cancer a sufficient criterion for family history? This question is crucial, not only from a clinical, cancer genetics viewpoint, but also when viewed in the context of $H$. pylori. In many family settings, previous studies have observed the intrafamilial presence of phylogenetically identical $H$. pylori strains (as assessed by rRNA gene patterns, called ribopatterns) [13]. Regardless of whether these findings are explained by person-to-person transmission or, alternatively, by the presence of a common source of infection, they collectively highlight the potential for intrafamilial transmission of H. pylori [13]. Mother-to-child and sibling-to-sibling transmission have also been reported [14]. Therefore, could this intrafamilial transmission of $H$. pylori strains contribute to the family history of gastric cancer?

On this basis, future studies considering the ties between a family history of gastric cancer and $H$. pylori eradication should also assess the molecular subtypes (e.g., analysis of CagA and the CagA-associated EPIYA polymorphisms [15]) and potential clonality of $H$. pylori strains, both in the examined individuals and in their first-degree affected relatives. Such study designs could ultimately contribute to a precision oncomicrobiology approach (for further description, see [1]) and could have farreaching implications.

H. pylori infection is a communicable disorder (i.e., an infectious disease), whereas gastric cancer is considered a chronic, non-communicable disease. Thus, the suggestion that intrafamilial transmission of $H$. pylori contributes to shared strain-induced common gastric cancer needs to be validated in independent settings before concrete clinical guidelines are formulated; nevertheless, the margins between the notion of communicable and non-communicable diseases would become looser if that scenario is validated. Besides, the idea that noncommunicable diseases may in fact be communicable is not novel; this notion has been previously suggested in different contexts and settings [16,17].

In conclusion, the study by Choi et al highlights that H. pylori eradication is of merit even in the presence of a family history of cancer; however, the methodological criteria implemented in this study should be viewed with caution 
before conclusive clinical guidelines are established. More importantly, clinical gastroenterologists should work closely with both clinical molecular geneticists and clinical molecular microbiologists to offer an overarching approach in clinical practice, by concurrently considering: (a) the family's genetic predisposition to gastric cancer; and (b) oncogenesis-related microbial genetic features of $H$. pylori strains isolated from these patients.

\section{References}

1. Mentis AA, Boziki M, Grigoriadis N, Papavassiliou AG. Helicobacter pylori infection and gastric cancer biology: tempering a double-edged sword. Cell Mol Life Sci 2019;76:2477-2486.

2. Sgouras D, Tegtmeyer N, Wessler S. Activity and functional importance of Helicobacter pylori virulence factors. Adv Exp Med Biol 2019;1149:35-56.

3. Uemura N, Okamoto S, Yamamoto S, et al. Helicobacter pylori infection and the development of gastric cancer. $N$ Engl J Med 2001;345:784-789.

4. Yamaoka Y, El-Zimaity HM, Gutierrez O, et al. Relationship between the cagA 3' repeat region of Helicobacter pylori, gastric histology, and susceptibility to low $\mathrm{pH}$. Gastroenterology 1999;117:342-349.

5. Rokkas T, Rokka A, Portincasa P. A systematic review and metaanalysis of the role of Helicobacter pylori eradication in preventing gastric cancer. Ann Gastroenterol 2017;30:414-423.

6. Yaghoobi M, Bijarchi R, Narod SA. Family history and the risk of gastric cancer. Br J Cancer 2010;102:237-242.
7. Choi IJ, Kim CG, Lee JY, et al. Family history of gastric cancer and Helicobacter pylori treatment. N Engl J Med 2020;382:427-436.

8. Rokkas T, Sechopoulos P, Pistiolas D, Margantinis G, Koukoulis G. Helicobacter pylori infection and gastric histology in firstdegree relatives of gastric cancer patients: a meta-analysis. Eur J Gastroenterol Hepatol 2010;22:1128-1133.

9. Mentis AA, Dardiotis E. Helicobacter pylori eradication for metachronous gastric cancer: an unsuitable methodology impeding broader clinical usage. Front Oncol 2019;9:90.

10. Wang K, Kan J, Yuen ST, et al. Exome sequencing identifies frequent mutation of ARID1A in molecular subtypes of gastric cancer. Nat Genet 2011;43:1219-1223.

11. Hansford S, Kaurah P, Li-Chang H, et al. Hereditary diffuse gastric cancer syndrome: CDH1 mutations and beyond. JAMA Oncol 2015;1:23-32.

12. Guilford P, Hopkins J, Harraway J, et al. E-cadherin germline mutations in familial gastric cancer. Nature 1998;392:402-405.

13. Georgopoulos SD, Mentis AF, Spiliadis CA, et al. Helicobacter pylori infection in spouses of patients with duodenal ulcers and comparison of ribosomal RNA gene patterns. Gut 1996;39:634-638.

14. Kivi M, Tindberg Y, Sörberg M, et al. Concordance of Helicobacter pylori strains within families. J Clin Microbiol 2003;41:5604-5608.

15. Sougleri IS, Papadakos KS, Zadik MP, Mavri-Vavagianni M, Mentis AF, Sgouras DN. Helicobacter pylori CagA protein induces factors involved in the epithelial to mesenchymal transition (EMT) in infected gastric epithelial cells in an EPIYA-phosphorylationdependent manner. FEBS J 2016;283:206-220.

16. Wainberg ML, Helpman L, Duarte CS, et al. Curtailing the communicability of psychiatric disorders. Lancet Psychiatry 2018;5:940-944.

17. Christakis NA, Fowler JH. The collective dynamics of smoking in a large social network. N Engl J Med 2008;358:2249-2258. 